\title{
Enhancing BPSK and OOK Modulations Using Hybrid 2BPSK- OOK Technique for Optical Fiber Communications Systems
}

\author{
Hossam Selmy ${ }^{1}$, Tawfik Ismail ${ }^{2}$, and Hossam M. H. Shalaby ${ }^{1}$ \\ ${ }^{1}$ Department of Electronics and Communications Engineering, Egypt-Japan University of Science and \\ Technology (E-JUST) \\ ${ }^{2}$ Department of Engineering Applications of Laser, National Institute of Laser Enhanced Science, \\ Cairo University
}

\begin{abstract}
A hybrid 2 slots binary phase shift keying-on off keying (hybrid 2BPSK-OOK) scheme is proposed as a new modulation technique to improve the performance of both conventional binary phase shift keying (BPSK) and on-off (OOK) modulation techniques in optical fiber communications systems. In conventional BPSK scheme, a consecutive stream of low power BPSK symbols are transmitted, while in the proposed scheme, a less number of high power BPSK symbols are transmitted in a two slot hybrid frame and their positions are exploited to transmit more bits. That is, the transmitted information is carried in both the positions and phases of the transmitted pulses. The transmission characteristics, transmitter and receiver block diagram, bandwidthutilization, and optimal decoding for the proposed scheme are all studied in this paper. An upper bound on the bit-error rate is obtained for the proposed scheme and is compared to that of the traditional schemes under the same energy per bit constraint. Our results reveal that, for both moderate and high signal-to-noise ratios, the proposed hybrid scheme achieves much lower levels of bit-error rates than that of ordinary coherent- and directdetected OOK schemes. However, at all energy per bit levels, the proposed scheme achieves less performance than that of ordinary BPSK. In addition, the proposed modulation scheme achieves much higher bandwidthutilization efficiencies as compared to that of ordinary schemes.
\end{abstract}

Keywords: binary phase shift keying modulation (BPSK), bit-error rate, hybrid modulations, on-off keying (OOK), optical fiber communications, symbol-error rate.

\section{INTRODUCTION}

Phase modulations represent important modulation category for both optical fiber and free space optical communications. Coherent detection of phase modulated signals increases the receiver sensitivity over traditional direct detection of intensity modulated signals. For both homodyne and heterodyne receivers, noticeable enhancements in the receiver sensitivity are achieved for systems limited by amplifier noise [1]. However, coherent detection of phase modulated signals requires much more complex receivers than that used for direct detection of intensity modulated signals.

On-off keying modulation technique represents an important type in the category of intensity modulations (IM) [2]. Historically, direct detection (DD) is commonly used in demodulating OOK signals. Recently, coherent detection (including both homodyne and heterodyne receivers) has been proposed to increase the receiver sensitivity [1]. For shot-noise limited systems, the sensitivity of heterodyne detection receivers is better by $3 \mathrm{~dB}$ than that of the direct detection receivers [3].

For further enhancements in the performances of ordinary phase and intensity modulations, hybrid modulations have been recently addressed. Both classical and quantum receivers for hybrid binary phase shift keying and binary pulse position modulation (BPSK-2PPM) scheme have been investigated and their performances have been compared to each other [4]. Another hybrid modulation scheme, which combines both PPM and binary phase shift keying subcarrier intensity modulation (BPSK-SIM) has been proposed [5]. This scheme offers BER performance that is superior to that of both BPSK-SIM and PPM at the same average transmitted power and bit rate. Furthermore, the hybridization of multi-pulse pulse-position modulation (MPPM) with BPSK has been recently proposed for high-speed optical fiber transmission [6].

In this paper, we aim at enhancing the performance of both ordinary BPSK and OOK modulations in optical fiber communications by proposing a new hybrid modulation scheme. By combing phase modulation with onoff keying modulation, the average transmitted power can be further reduced, resulting in relatively high bandwidth-utilization efficiency at a reasonable receiver sensitivity. Specifically, we propose hybrid 2-slots frames in which data bits are encoded using both positions and phases of transmitted optical pulses. Also, coherent detection is used for demodulating both BPSK and OOK symbols in the hybrid frame. We call the proposed scheme hybrid 2 slots binary phase shift keying-on off keying (hybrid 2BPSK-OOK) modulation.

The rest of the paper is organized as follows: In Section 2, we develop a general system model for hybrid 2BPSK-OOK scheme and study its transmission characteristics. Also, the differences between the proposed hybrid scheme and both ordinary BPSK and OOK schemes are stated. In Section 3, we present the transmitter and receiver block diagram for the hybrid scheme. Also, we state its maximum-likelihood decoding (optimal decoding) in optically pre-amplified channels with amplifier-noise limited case. The performance evaluation for the hybrid scheme is derived in Section 4. The numerical simulations that indicate the performance comparisons with ordinary schemes are investigated in Section 5. Finally, the conclusion is given in Section 6. 


\section{PROPOSED SYSTEM MODEL AND CHARACTERISTICS}

Here, we propose a hybrid 2BPSK-OOK modulation scheme in which binary phase shift keying is combined with on-off keying modulation in 2-slots hybrid frames. The goal of proposing this hybrid scheme is to enhance the performance of both ordinary BPSK and OOK modulation schemes used for optical fiber communications systems. Generally, instead of transmitting a consecutive stream of optical pulses (each with a relatively small power) as in ordinary BPSK scheme, we propose to transmit less number of high-power BPSK pulses and exploit the positions of these pulses to transmit more bits in the hybrid frame. Furthermore, more bits would be encoded in the phases of the transmitted optical pulses in the hybrid frames.

Specifically, for a hybrid frame of size $M$ slots, we could transmit any combination of signal and non-signal slots during these $M$ slots and each transmitted optical pulse is encoded with one of two binary phases $0^{\circ}$ or $180^{\circ}$. The possible combinations of the slots (available symbols) and the hybrid frame structure for $M=2$ are indicated in Fig. 1.

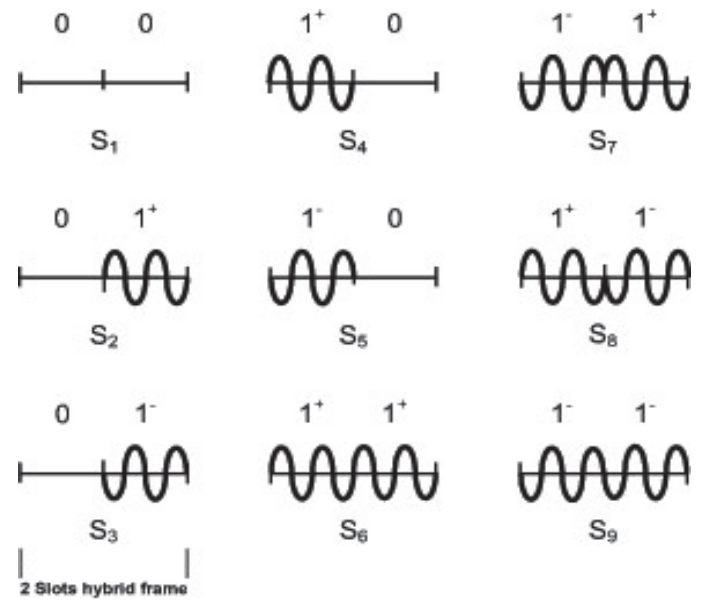

Figure 1. Symbol set and hybrid frame structure for $M=2$.

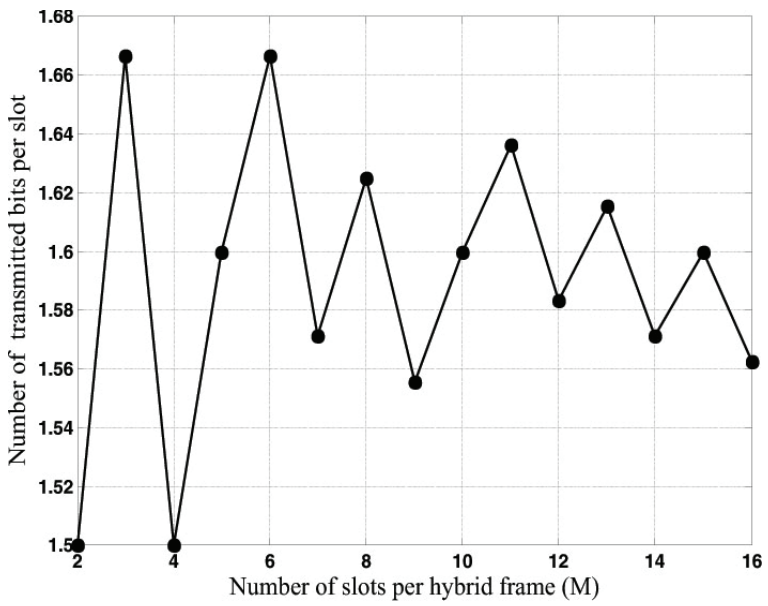

Figure 2. Number of transmitted bits per slot.

For this case, the set of the available symbols contains 9 symbols starting with the symbol that has two nonsignal slots and ending with the symbols that have two signal slots. Using these symbols, 3 bits could be easily encoded in the transmitted 2-slots hybrid frames.

Generally, for hybrid M-slots frame, the number of available symbols in the system is given by

$$
\text { Number of symbols }=\sum_{i=0}^{M} 2^{i}\left(\begin{array}{c}
M \\
i
\end{array}\right)
$$

Clearly, number of available symbols and consequently number of transmitted bits per frame are increased by increasing the hybrid frame size (increasing M). However, for fair comparison between hybrid schemes (with different frame sizes) and both BPSK and OOK schemes, the number of transmitted bits per slot should be considered. This is given by

$$
\text { Bits per slot }=\frac{1}{M}\left\lfloor\log _{2}\left(\sum_{i=0}^{M} 2^{i}\left(\begin{array}{c}
M \\
i
\end{array}\right)\right)\right\rfloor
$$

For both ordinary BPSK and OOK schemes, the number of transmitted bits per slot is one bit. However, using the proposed hybrid scheme, significant increases in this number can be achieved. Figure 2 shows the number of transmitted bits per slot at different sizes of the hybrid frame. At $M=2$ slots, number of transmitted bits per slot is 1.5 bits which represents an increase in the transmission rate by $50 \%$ than that of both ordinary BPSK and OOK schemes. However, as indicated from the figure, increasing size of hybrid frame does not always result in increasing number of transmitted bits per slot. Clearly with $M=4$ slots, the number of transmitted bits per slot is 1.5 bits, which is the same as the case of $M=2$ slots.

Also, a maximum value of 1.667 bits per slot occurs at $M=3$ and $M=6$. Obviously, this peak value does not represent a significant increase over the case of $M=2$. Therefore, toward reducing the BER and simplify the structures of both encoder and decoder, the selection of $M=2$ slots is considered throughout this paper.

The bit encoding for hybrid 2BPSK-OOK scheme is somehow tricky and generally there is no known optimum method to encode such scheme, the same as the case of encoding multi-pulse pulse-position modulation (MPPM) symbols [7]. The simplest, however inefficient, encoding algorithm for this scheme is to select only 8 symbols out of the available 9 symbols to be used for transmission and discarding the remaining symbol. In this case, each transmitted symbol is encoded by three data bits.

Obviously, not all available symbols are used for transmission and there exists an optimal bit-symbol mapping that results in the lowest BER. Another simple and more efficient encoding algorithm is to create compound 
symbols by cascading enough hybrid symbols to ensure that the total number just exceeds a power of 2 and then encoding suitably larger blocks of information bits into this compound signal set [8]. However, the bit encoding and decoding for hybrid 2BPSK-OOK are not in the scope of this paper, they may represent important design issues that could be considered in future research works.

\section{TRANSMITTER AND RECEIVER BLOCK DIAGRAM AND DECODING ALGORITHM}

The block diagram of the transmitter and receiver of hybrid 2BPSK-OOK system is shown in Fig. 3 . At the transmitter side, the transmitted data bits are first fed to symbol encoder, which maps fixed length sequences of input data bits into the corresponding two-slot symbols of the available set. The signal and non-signal slots of each symbol are generated by intensity modulating a continuous wave $(\mathrm{CW})$ laser source, while, the phase associated with each signal slots is generated using optical binary phase modulator. Mach-Zehnder configurations are used in both intensity and phase modulators. At the receiver side, coherent detection (for both OOK and BPSK schemes) is adopted to increase the receiver sensitivity. In addition, the coherent receiver implements balanced optical detectors in order to minimize the noise generated from local oscillator (LO).

Generally, for coherent receivers limited by optical amplifier noise, both heterodyne and homodyne detection receivers achieve same performance [1]. The photodetector output of the homodyne receiver (or the IF demodulator output of the heterodyne receiver) is then fed to a slot integrator. At the end of each slot, the slot integrator output is squared and both the integrator output and its square value (slot energy) are compared with zero and certain threshold, respectively. The comparison results are passed to a DSP to determine the received symbol. If the slot energy is greater than the threshold, the slot is decoded as signal slot; otherwise it is decoded as non-signal slot. For signal slots, the output of the integrator is compared with zero, if it is larger, the associated phase is decoded as $0^{\circ}$; otherwise, and it is decoded as $180^{\circ}$. After decoding the two slots of the hybrid frame, the determined symbol is then fed to symbol decoder that produces the corresponding output bits.

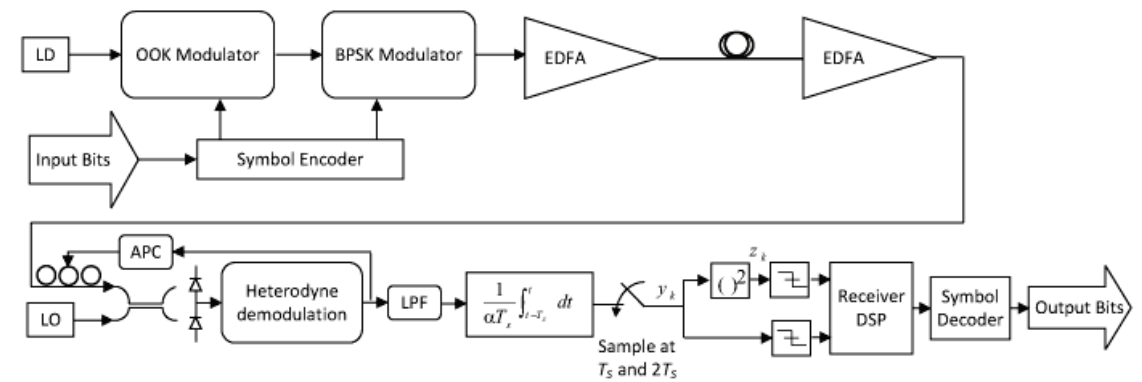

Figure 3. Transmitter and receiver block diagram for hybrid 2BPSK-OOK scheme.

\section{Decoding Algorithm}

1) Perform coherent detection for received hybrid frame by homodyne or heterodyne demodulation.

2) Get received signal amplitude in each slot.

3) Determine the energy received in each slot by squaring the integrator output samples.

4) Determine the signal slots by comparing the received slots energies to a specific threshold.

5) Determine the encoded phases of the predetermined signal slots by comparing their integrator samples to zero.

\section{PERFORMANCE EVALUATION}

In this section we aim at evaluating the bit-error rate for the proposed hybrid 2BPSK-OOK scheme $\left(B E R_{\mathrm{H}}\right)$. In this evaluation, the optical fiber is considered as the transmission medium and the fiber span is assumed to have many optical amplifiers so that the system is limited by the amplified spontaneous emission (ASE) noise generated from these amplifiers [1]. The input data stream is divided into blocks, each is encoded in 2-slot hybrid frame. The symbols set contains nine symbols. The input data is assumed to be uncoded, i.e., the numbers of data ones and zeroes are equal and the probability of using each symbol is the same for all symbols. The symbol-error rate $S E R_{H}$ for the hybrid scheme is then given by:

$$
\begin{aligned}
S E R_{\mathrm{H}} & =1-P_{\mathrm{C}} \\
& =1-\left(1-B E R_{\text {on-off }}\right)^{2}\left[\frac{1}{9}+\frac{4}{9}\left(1-B E R_{\mathrm{BPSK}}\right)+\frac{4}{9}\left(1-B E R_{\mathrm{BPSK}}\right)^{2}\right] \\
& =1-\left(1-B E R_{\text {on-of }}\right)^{2}\left(1-\frac{2}{3} B E R_{\mathrm{BPSK}}\right)^{2}
\end{aligned}
$$

where $\mathrm{P}_{\mathrm{C}}$ is probability of correct decoding for the received symbol, $B E R_{\mathrm{on} \text {-off }}$ and $B E R_{\mathrm{BPSK}}$ are bit-error rates for ordinary on-off and BPSK schemes, respectively. Generally, it is difficult to get a closed form for $B E R_{\mathrm{H}}$. 
However, in the hybrid scheme that transmits $L$ bits per frame, where $L$ is an integer number (in our case $L=3$ bits), the relation between $B E R_{\mathrm{H}}$ and $S E R_{\mathrm{H}}$ is upper bounded by [9]:

$$
B E R_{H} \leq \frac{2^{L-1}}{2^{L}-1} \times S E R_{H}
$$

Here, coherent detection is used instead of direct detection in demodulating the proposed hybrid scheme. However, in the analysis of preamplifiered coherent receivers there are many types of noise sources.

The main three noise sources are the preamplifiered spontaneous emission noise (ASE), the local oscillator noise, and the detector noise, which includes both shot and thermal noises. Here, we use a dual-photodiode balanced receiver to reduce the LO noise and increase the signal power. Also, we assume that the detector shot noise variance is much larger than that of the thermal noise so that the detector thermal noise can be neglected. Moreover, to simplify the receiver structure and for optimal signal mixing, the polarization of the received signal is aligned to that of the LO laser using automatic polarization control APC as indicated in Fig. 3.

We recall the general model for coherent detection of optical phase modulated signals to develop the coherent detection model for the proposed hybrid scheme. The bit error rate for both $\mathrm{BER}_{\text {on-off }}$ and $\mathrm{BER}_{\mathrm{BPSK}}$ in the hybrid frame could be obtained in similar procedures mentioned in [1] and [10]. Substituting these results into $\mathrm{SER}_{\mathrm{H}}$, we obtain an upper bound on $\mathrm{BER}_{\mathrm{H}}$ for hybrid 2BPSK-OOK scheme.

\section{NUMERICAL RESULTS}

In this section we compare the performance achieved by hybrid 2BPSK-OOK with that of ordinary BPSK and OOK modulation schemes. The comparison is carried for bit-error rate at the same value of energy per bit $E_{\mathrm{b}}$. Although, the comparison is performed for optical fiber channels, it could be extended for other optical channels by considering the appropriate optical transmission model. The noise considered in the simulations is assumed to be dominated by optical amplifier ASE noise. The noise is modeled as Gaussian noise with zero mean and variance of $\sigma_{\mathrm{n}}^{2}$ clearly, the modulation scheme that achieves higher performance at the same $E_{\mathrm{b}}$ level is highly desired in this case. However, one of the main advantages of the proposed hybrid scheme is its higher transmission rate which is about 1.5 times the transmission rate of both BPSK and OOK modulations.

The energy per bit for ordinary BPSK, OOK and hybrid 2BPSK-OOK schemes can be expressed as $E_{\mathrm{bB}}=A_{\mathrm{B}}^{2} \cdot T_{\mathrm{b}}, E_{\mathrm{bO}}=1 / 2 \cdot A_{\mathrm{O}}{ }^{2} \cdot T_{\mathrm{b}}$, and $E_{\mathrm{bH}}=4 / 9 \cdot A_{\mathrm{H}}^{2} \cdot T_{\mathrm{b}}$, respectively, where $A_{\mathrm{B}}, A_{\mathrm{O}}$, and $A_{\mathrm{H}}$ are the amplitudes of demodulated signals for ordinary BPSK, OOK, and hybrid 2BPSK-OOK schemes, respectively. Here $T_{\mathrm{b}}$ is the bit duration for both BPSK and OOK signals and is the same as the slot duration for the hybrid scheme. Clearly, under equal energy per bit constraint, we have $E_{\mathrm{bB}}=E_{\mathrm{bO}}=E_{\mathrm{bH}}$ and $A_{\mathrm{B}}{ }^{2}=(1 / 2) A_{\mathrm{O}}{ }^{2}=(4 / 9) \mathrm{A}_{\mathrm{H}}{ }^{2}$. In this case, the ratio between the received energy per bit to slot noise energy $E_{\mathrm{b}} / N_{0}$ for all schemes can be written as:

$$
\frac{E_{\mathrm{b}}}{N_{0}}=\frac{A_{\mathrm{B}}^{2}}{\sigma_{\mathrm{n}}^{2}}=\frac{\frac{1}{2} A_{\mathrm{O}}^{2}}{\sigma_{\mathrm{n}}^{2}}=\frac{\frac{4}{9} A_{\mathrm{H}}^{2}}{\sigma_{\mathrm{n}}^{2}}
$$

Figure 4 indicates the performance comparison between the proposed hybrid scheme and the ordinary BPSK scheme under same energy per bit constraint. The comparison is carried in terms of achieved bit-error rate versus the received energy per bit to noise energy per slot ratio. Clearly, at all values of the received energy per bit to noise energy per slot ratio, BPSK scheme performs better than the proposed hybrid scheme, i.e., achieves less BER. However, the hybrid scheme still outperform the BPSK in terms of the transmission rate. Clearly, the transmission rate of the hybrid scheme is one and half times the transmission rate of ordinary BPSK. However, to achieve the same BER performance, a power penalty could be transmitted for the hybrid scheme. Numerically, at a $B E R=10^{-8}$, a penalty of $2 \mathrm{~dB}$ in $E_{\mathrm{b}}$ is required. Also, the performance gap between the two schemes is much increased at higher levels of $E_{\mathrm{b}} / N_{0}$. This performance gap rises from the decoding procedures associated with the two schemes. Clearly, polarity decoding is used for BPSK, while both polarity and square value (energy decoding) of the integrator output is used for the hybrid frame. Generally, energy decoding results in much decoding errors even that the signal amplitude for the hybrid scheme is higher than that of the BPSK.

Figure 5 shows a performance comparison between the proposed hybrid scheme and the ordinary OOK schemes (both coherent and direct detected). Again the comparison is carried under the same energy per bit level and the system noise is assumed to be dominated by optical amplifier ASE noise. In the figure, the bit error rates are plotted versus the received energy per bit to noise energy per slot ratio. Clearly, at all levels of $E_{\mathrm{b}} / N_{0}$ the hybrid scheme outperforms the direct detected OOK scheme in terms of the achieved BER. However, at small values of $E_{\mathrm{b}} / N_{0}$, the coherent detected OOK achieves slightly better performance than that of the hybrid scheme. In contrast, the proposed hybrid scheme results in lower BER levels at high values of $E_{\mathrm{b}} / N_{0}$. Furthermore, the performance gap between the hybrid schemes and ordinary ones is increased by raising the level of $E_{\mathrm{b}} / N_{0}$. Specifically, at $B E R=10^{-6}$, the proposed scheme achieves a reduction in the required $E_{\mathrm{b}}$ by $0.5 \mathrm{~dB}$ and $2 \mathrm{~dB}$ than that of coherent and direct detected OOK schemes, respectively. Clearly, coherent detection results in a better receiver sensitivity than direct detection, but it requires more complex receiver structure. Although, the proposed hybrid scheme could achieves nearly the same performance of the coherent detected OOK, it still increases the transmission rate by a percentage of $50 \%$. Obviously, with the same complexity in transmitter and 
receiver structures, this enhancement in the transmission rate has an important practical consideration.

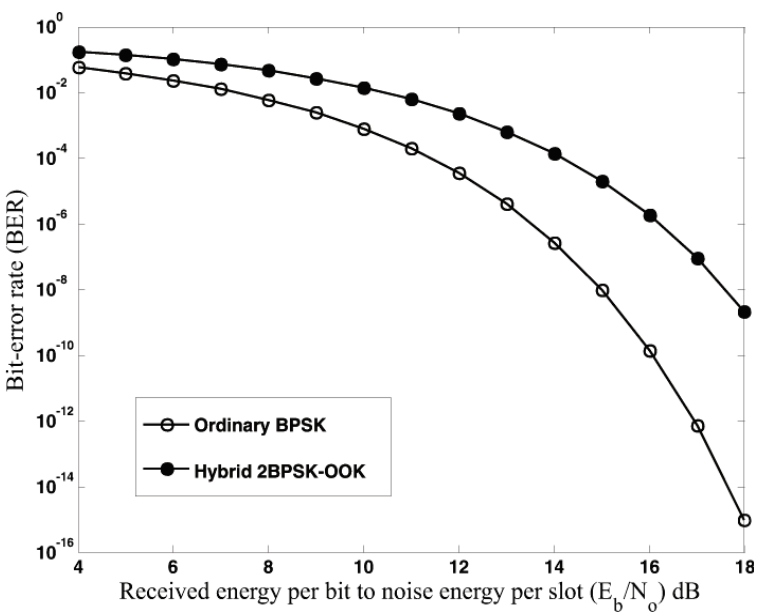

Figure 4. Bit-error rate versus received energy per bit to noise energy per slot ratio for BPSK and hybrid schemes.

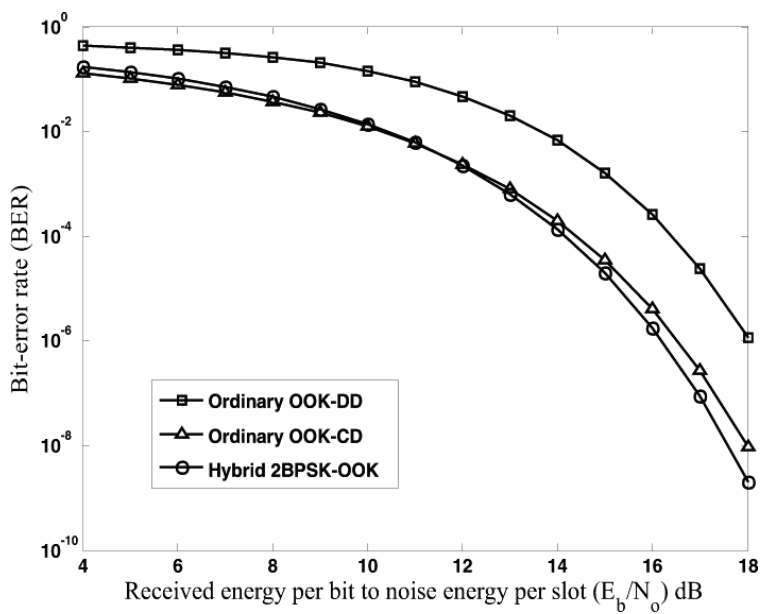

Figure 5. Bit-error rate versus received energy per bit to noise energy per slot ratio for $O O K$ and hybrid schemes.

\section{CONCLUSION}

A new hybrid modulation scheme, hybrid 2BPSK-OOK, has been proposed for optical fiber communication systems. The proposed scheme combines both BPSK and OOK modulations in one unified hybrid scheme. The proposed scheme achieves much higher bandwidth utilization efficiency than that of the ordinary schemes. Both the transmitter and receiver structures along with the optimal decoding algorithm have been presented and discussed for optical fiber channel systems. An upper bound for the bit-error rate of the proposed scheme has been derived taking into account the effect of optical amplifiers noise. Intensive numerical evaluations have been performed to compare the performance achieved by the proposed hybrid scheme with that of both BPSK and OOK schemes. The numerical evaluations have been carried out under same energy per bit constraint. Our results indicate the superior performance of the proposed hybrid scheme under same energy per bit constraint over both coherent and direct detection OOK schemes. In contrast, the results indicate that the proposed scheme achieves worse performance than that of ordinary BPSK.

\section{REFERENCES}

[1] K.-P. Ho, "Phase-Modulated Optical Communication Systems," Springer, New York, 2005.

[2] D. Ben-Eli, Y.E. Dallal, and S. Shamai, "Performance bounds and cut-off rates of quantum limited OOK with optical amplification," IEEE Journal on Selected Areas in Communications, vol. 13, no. 3, pp. 510-530, Apr. 1995.

[3] M.M. Fernandez and V.A. Vilnrotter, "Coherent optical receiver for PPM signals under atmospheric turbulence," IEEE Aerospace Conference, pp. 1604-1611, Mar. 2005.

[4] V.A. Vilnrotter and C. Lau, "Quantum detection and channel capacity for communications applications," Proc. SPIE 4635, pp. 103-115 (2002).

[5] M. Faridzadeh, A. Gholami, Z. Ghassemlooy, and S. Rajbhandari, "Hybrid PPM-BPSK subcarrier intensity modulation for free space optical communications," in Proc. 16th European Conference on Networks and Optical Communications (NOC 2011), pp. 36-39, Jul. 20-22, 2011.

[6] H. Selmy, H.M.H. Shalaby, and Z. Kawasaki, "Proposal and performance evaluation of a hybrid BPSKmodified MPPM technique for optical fiber communications systems, "Journal of Lightwave Technology, vol. 31, no. 22, pp. 3535-3545, Nov. 2013.

[7] S. Liu and F.R. Kschischang, "Coding for MPPM-like systems," in Proc. 25th Biennial Symposium on Communications (QBSC 2010), Kingston, Ontario, Canada, 12-14 May 2010.

[8] M. Simon and V. Vilnrotter, "Performance analysis and tradeoff for dualpulse PPM on optical communications channels with direct detection," IEEE Trans. Commun., vol. 52, no. 11, pp. 1969-1979, Nov. 2004.

[9] N. Aoki, T. Ohtsuki, and I. Sasase, "Performance analysis of multi-pulse pulse position modulation using avalanche photodiode in optical intersatellite links," IEICE Trans. Commun., vol. E79-B, no. 1, pp. 52-56, Jan. 1996.

[10] J. G. Proakis, "Digital Communications,” 4th ed., Mc-GrawHill, Boston, MA, 2001. 